\title{
Doublel-robot coordination workspace Analysis based on Matlab
}

\author{
Wei Wang, Yong Xu \\ School of Mechanical and Automotive Engineering, Shanghai University of Engineering and Technology, Shanghai, 201620
}

\begin{abstract}
Aiming at the requirements of dual robot collaborative operation, a dual robot cooperation system model is established in SolidWorks2012 software to study the dual robot cooperation space. The $\mathrm{D}-\mathrm{H}$ parameters are established, and the kinematics positive solution equation is obtained. The dual robot cooperative kinematics model is given. Based on the Monte Carlo method, the workspace of the dual robot is solved. The extreme value theory method is used to analyze and calculate, so as to extract the precise boundary contour of the common area of the dual robot workspace, and the collaborative space boundary surface and limit position of the dual robot are determined. The optimal coordinated working space of the dual robot end effector is obtained, which lays a theoretical foundation for the coordinated trajectory planning of the dual robot.
\end{abstract}

\section{Introduction}

The research on the workspace problem of multi-robot collaborative work is one of the difficult problems in the research of multi-robot system ${ }^{[1]}$. Collaborative space is a common area that can be achieved by multiple robots (represented by dual robots). It is an important kinematic indicator for measuring the working ability of dual robots ${ }^{[2]}$. Comprehensive research literature at home and abroad has made little research on the collaborative space of dual robots, mostly focusing on single-industry robots, less degrees of freedom, and working spaces of parallel robots ${ }^{[3]}$.The research of collaborative workspace has a very important role in the design, path planning, collision avoidance research and optimal operation configuration of dual robots ${ }^{[4]}$.

In this paper, Monte Carlo method is used. Monte Carlo is a numerical method for solving mathematical problems by means of random sampling. This method is easy to implement computer graphics display function, and the calculation speed is fast and the error and dimension are irrelevant ${ }^{[6]}$. The collaborative workspace of the dual robot collaborative operation system is analyzed ${ }^{[7]}$.

\section{Modeling of dual robot collaborative operation system}

A two-robot collaborative system consisting of two six-degree-of-freedom industrial robots is the research object. The system model adopts the layout of the main robot 1 (left) and the slave robot 2 (right), and the distance between the two robots is $2 \mathrm{~L}$. The structural model of the dual robot collaborative operation system is shown in Figure 1.
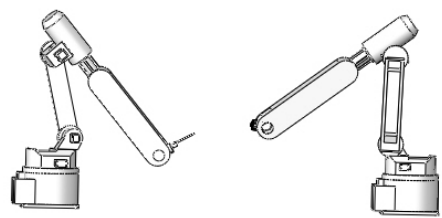

Figure 1. Structural model of a dual robot

\section{Kinematics analysis of robots}

\subsection{Establishment of coordinate system and determination of $\mathrm{D}$-H parameters}

The description of the kinematics of the robot requires two coordinate systems ${ }^{[5]}$. One is the local coordinate system of a single robot. The coordinate system representing the main robot is $\mathrm{O}_{1} \mathrm{X}_{1} \mathrm{Y}_{1} \mathrm{Z}_{1}$, which represents the coordinate system of the slave robot is $\mathrm{O}_{2} \mathrm{X}_{2} \mathrm{Y}_{2} \mathrm{Z}_{2}$; the other is global. The coordinate system OXYZ, the local coordinate system of the master robot and the slave robot are symmetric about the global coordinate system. Figure 2 is the D-H coordinate system of the dual robot.

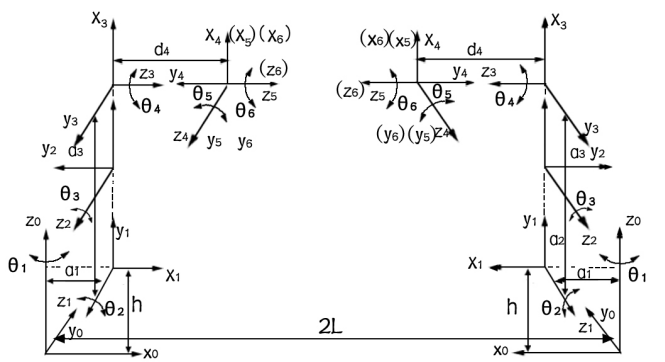

Figure 2. D-H coordinate system of the dual robot cooperation system 
Table 1. Dual robot cooperation system D-H parameter table (Note: $\mathbf{a} 1=255 \mathrm{~mm}, a 2=885 \mathrm{~mm}, a 3=35 \mathrm{~mm}, d 4=980 \mathrm{~mm}$, $2 \mathrm{~L}=3000 \mathrm{~mm}$ )

\begin{tabular}{|c|c|c|c|c|}
\hline$i$ & $\alpha$ & $a_{i-1}$ & $d_{i}$ & $\theta_{i}$ \\
\hline 1 & 0 & 0 & 0 & $(-180,180)$ \\
\hline 2 & -90 & 255 & 0 & $(-155,45)$ \\
\hline 3 & 0 & 885 & 0 & $(-160,120)$ \\
\hline 4 & -90 & 35 & 980 & $(-350,350)$ \\
\hline 5 & 90 & 0 & 0 & $(-140,140)$ \\
\hline 6 & -90 & 0 & 0 & $(-345,345)$ \\
\hline
\end{tabular}

\subsection{Kinematics positive solution}

The kinematic positive solution is to determine the position of the end pose based on the value of the known joint variable. The homogeneous coordinate transformation between adjacent joints is sequentially performed by the formula (1), and the end effector is multiplied by the formula (2) to obtain the end effector relative to the base coordinate system. coordinate. The kinematics positive solution is also solved by the kinematics positive solution formula ${ }^{[8]}$.

The link transformation matrix is:

$$
{ }_{i}^{i-1} T=\left[\begin{array}{cccc}
c_{i} & -s_{i} & 0 & \alpha_{i-1} \\
s_{i} c_{\alpha_{i-1}} & c_{i} c_{\alpha_{i-1}} & -\sin \alpha_{i-1} & -s_{\alpha_{i-1}} d_{i} \\
s_{i} s_{\alpha_{i-1}} & c_{i} s_{\alpha_{i-1}} & c_{i} \alpha_{i-1} & c_{\alpha_{i-1}} d_{i} \\
0 & 0 & 0 & 1
\end{array}\right]
$$

$$
\text { formula: } \begin{aligned}
& c_{i}=\cos \theta_{i} ; s_{i}=\sin \theta_{i} ; c \alpha_{i-1}=\cos \alpha_{i-1} ; \\
& s \alpha_{i-1}=\sin \alpha_{i-1}
\end{aligned}
$$

It can be seen from Table 1 that the values of the corresponding parameters are substituted:

$$
\begin{aligned}
& { }_{1}^{0} T=\left[\begin{array}{cccc}
c_{1} & -s_{1} & 0 & 0 \\
s_{1} & c_{1} & 0 & 0 \\
0 & 0 & 1 & 0 \\
0 & 0 & 0 & 1
\end{array}\right] \quad{ }_{2}^{1} T=\left[\begin{array}{cccc}
c_{2} & -s_{2} & 0 & a_{1} \\
0 & 0 & 1 & 0 \\
-s_{2} & c_{2} & 0 & 0 \\
0 & 0 & 0 & 1
\end{array}\right]
\end{aligned}
$$

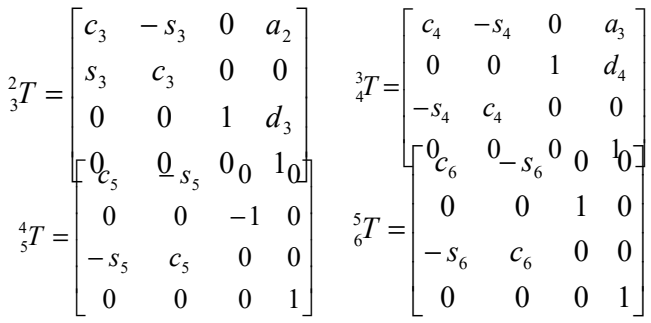
(2):

Solved by the kinematics positive solution formula

$$
{ }_{6}^{0} T={ }_{1}^{0} T_{2}^{1} T_{3}^{2} T_{4}^{3} T_{5}^{4} T_{6}^{5} T
$$

$$
{ }_{6}^{0} T=\left[\begin{array}{cccc}
n_{x} & o_{x} & a_{x} & p_{x} \\
n_{y} & o_{y} & a_{y} & p_{y} \\
n_{z} & o_{z} & a_{z} & p_{z} \\
0 & 0 & 0 & 1
\end{array}\right]
$$

Substituting each motion parameter into equation (3) can obtain the position vector of the robot's end in the relative coordinate system in the reference coordinate system:

$$
\left[\begin{array}{c}
p_{x} \\
p_{y} \\
p_{z}
\end{array}\right]=\left[\begin{array}{c}
c_{1}\left(a_{2} c_{3}+a_{3} c_{23}-d_{4} s_{23}\right)-a_{1} s_{1} \\
s_{1}\left(a_{2} c_{3}+a_{3} c_{23}-d_{4} s_{23}\right)+a_{1} s \\
-a_{3} s_{23}-a_{2} s_{2}-d_{4} c_{23}
\end{array}\right]
$$

As can be seen from (4), the position coordinates of the robot end effector are known in the case where the various parameters of the robot are known.

Therefore, as long as the set of all the points in the formula (4) is satisfied, the working space of the single robot is constructed, and thus the set $\mathrm{P}$ of the points satisfying the robot working space can be established as follows:

$$
p=\left\{\begin{array}{ccc}
x=f_{x}\left(\theta_{1}, \theta_{2}, \theta_{3}\right) & \theta_{\min } \leq \theta_{t} \leq \theta_{\max } \\
(x, y, z) & y=f_{y}\left(\theta_{1}, \theta_{2}, \theta_{3}\right) \\
z=f_{z}\left(\theta_{1}, \theta_{2}, \theta_{3}\right) & t=1,2,3
\end{array}\right\}
$$

\section{Solution and Simulation of Double Robot Collaboration Space}

\subsection{Derivation of Mathematical Model Equations for Dual Robot Cooperative Workspace}

From the point set formula (5), the end trajectory workspace of a single robot in the local coordinate system can be obtained, The working space set of the main robot 1 is defined as $\mathrm{p} 1$, and the working space of the robot 2 is set to $\mathrm{p} 2$, and the working space in the local coordinate system is down-converted to the world coordinate system according to the formula (5), and the main robot 1 and the slave robot 2 are obtained. The working space equation in the world coordinate system is:

The set of workspace points of the master robot 1 is:

$$
p^{1}=\left\{\begin{array}{ccc}
x^{1}=f_{x}^{1}\left(\theta_{1}^{1}, \theta_{2}^{1}, \theta_{3}^{1}\right) & \\
y^{1}=f_{y}^{1}\left(\theta_{1}^{1}, \theta_{2}^{1}, \theta_{3}^{1}\right) & \theta_{\min }^{1} \leq \theta_{t}^{1} \leq \theta_{\max }^{1} \\
z^{1}=f_{z}^{1}\left(\theta_{1}^{1}, \theta_{2}^{1}, \theta_{3}^{1}\right)-L & t=1,2,3
\end{array}\right\}(6)
$$

Similarly, the working space equation from Robot 2 is:

$$
p^{2}=\left\{\begin{array}{ccc}
x^{2}=f_{x}^{2}\left(\theta_{1}^{2}, \theta_{2}^{2}, \theta_{3}^{2}\right) \\
\left(x^{2}, y^{2}, z^{2}\right) & y^{2}=f_{y}^{2}\left(\theta_{1}^{2}, \theta_{2}^{2}, \theta_{3}^{2}\right) \\
z^{2}=f_{z}^{2}\left(\theta_{1}^{2}, \theta_{2}^{2}, \theta_{3}^{2}\right) & \theta_{\min }^{2} \leq \theta_{t}^{2} \leq \theta_{\max }^{2} \\
& t=1,2,3
\end{array}\right\} \text { (7) }
$$

The simultaneous (6), (7) can establish the workspace set $\mathrm{M}$ of the common area of the dual robot cooperative welding system, that is as:

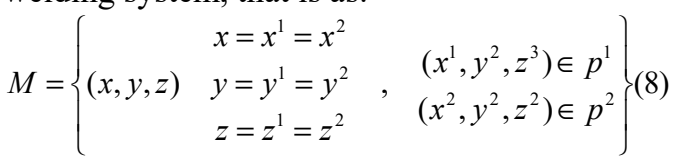

\subsection{Simulation analysis of dual robot collaborative space}


The workspace cloud map is established according to Monte Carlo numerical analysis. First, in the Matlab environment, use the random jointvariable:

$$
\theta_{i}=\theta_{i} \min +\left(\theta_{i} \max -\theta_{i} \min \right) \times \operatorname{Rand}(N, 1), i=1 \sim 3
$$

Discrete the joint angles of the two robots, Then, according to the positive kinematics equation (4) and the intersection set of the two-robot cooperative space points (8), the spatial coordinate value of the end effector, that is, the workspace [8] is obtained. The superimposed area is a common area of the dual robot working space, that is, a collaborative space. As shown in Figure (3).

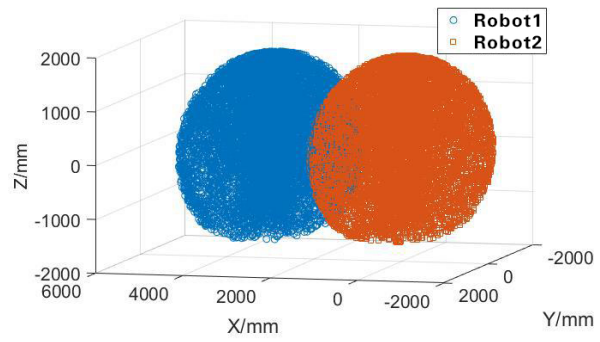

(Figure a).Collaborative 3D workspace

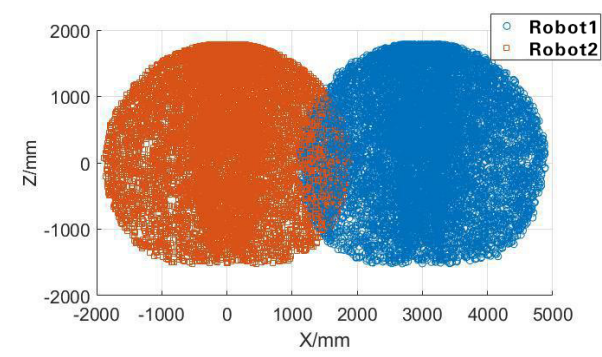

(Figure b).Collaborative workspace XOY plane projection

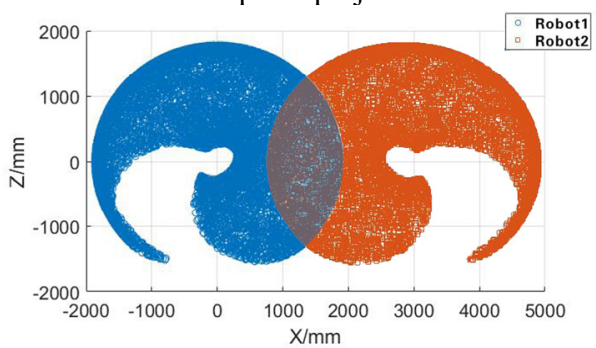

(Figure c).Cross-sectional view of the collaborative space $\mathrm{XOZ}$

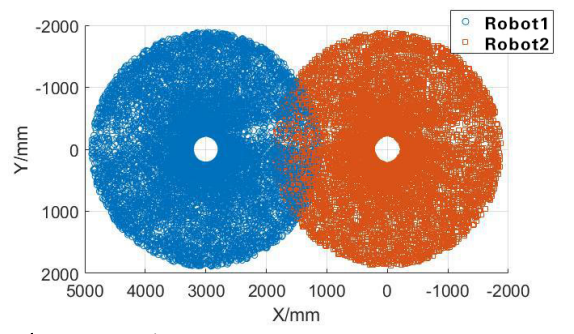

（Figure d) . Collaborative workspace XOZ plane

Figure 3. collaborative workspace diagram

From Fig. (3), the working limit range and the optimal working space limit range of the dual robot in the three coordinate systems of $\mathrm{X}, \mathrm{Y}$ and $\mathrm{Z}$ can be obtained. The size of the workspace is related to the robot's structural parameters, which are also related to the assembly position of the two robots.

\subsection{Drawing the boundary curve of the collaborative space and analyzing the numerical results}

In order to obtain the boundary curve of the collaborative workspace [6], the boundary value curve of the collaborative workspace is extracted by the extreme value theory and the search region method ${ }^{[10]}$. The graph of the two-dimensional workspace is shown in Figure (4).The common area is the boundary outline of the collaborative workspace., as shown in Figure 5.

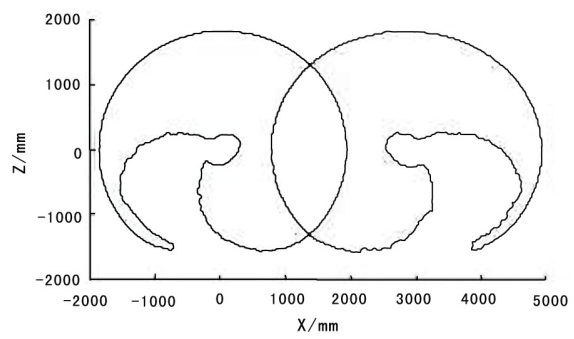

Figure 4. Two-robot collaborative space two-dimensional boundary map

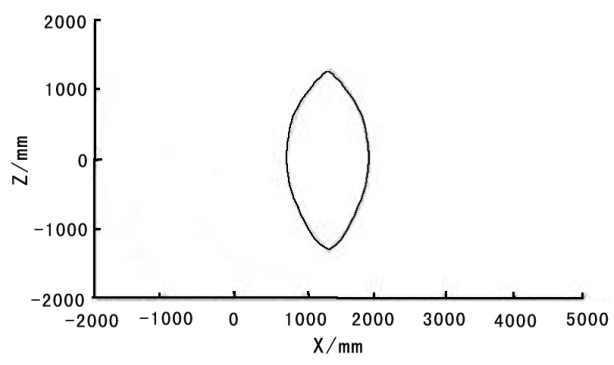

Figure 5. Shape of collaborative space

From the above solution method, the spatial region of the dual robot cooperative workspace and the key points of the extreme position and joint angle are solved based on the Matlab numerical solution method. The results are shown in Table 2 - Table 4.

Table 2. Single Robot Workspace Limit Position and Joint Variable Parameters

\begin{tabular}{|l|c|c|c|c|}
\hline & $\begin{array}{c}\text { Extreme-po } \\
\text { sition/mm }\end{array}$ & $\theta_{1} / \mathrm{rad}$ & $\theta_{2} / \mathrm{rad}$ & $\theta_{3} / \mathrm{rad}$ \\
\hline $\mathrm{X}_{\max }$ & 1872.67 & 0.6786 & -0.4586 & -1.5663 \\
$\mathrm{X}_{\min }$ & -1870.46 & -1.7753 & -0.0843 & -2.2175 \\
$\mathrm{Y}_{\max }$ & 1880.29 & -1.0753 & -2.4059 & 0.444 \\
$\mathrm{Y}_{\min }$ & -1880.29 & 2.8471 & -2.9142 & -2.1889 \\
$\mathrm{Z}_{\max }$ & 1880.38 & 0.0109 & -1.3511 & -1.2585 \\
$\mathrm{Z}_{\min }$ & -1880.38 & 0.1097 & -1.6486 & 0.1557 \\
\hline
\end{tabular}

Table 3. Collaborative workspace limit position and master robot 1 joint variable parameters

\begin{tabular}{|c|c|c|c|c|}
\hline & $\begin{array}{c}\text { Extreme-po } \\
\text { sition/mm }\end{array}$ & $\theta_{1}^{1} / \mathrm{rad}$ & $\theta_{1}^{2} / \mathrm{rad}$ & $\theta_{1}^{3} / \mathrm{rad}$ \\
\hline
\end{tabular}




\begin{tabular}{|c|c|c|c|c|}
\hline $\mathrm{X}_{\max }$ & 2102.56 & 1.6466 & -1.8479 & -1.8479 \\
$\mathrm{X}_{\min }$ & 900.872 & -0.8271 & 0.0521 & 0.0521 \\
$\mathrm{Y}_{\max }$ & 1112.33 & 1.7758 & 0.4004 & 0.4004 \\
$\mathrm{Y}_{\min }$ & -1112.33 & 0.5134 & -2.8906 & -2.8906 \\
$\mathrm{Z}_{\max }$ & 1290.78 & 0.9147 & -0.5308 & -0.5308 \\
$\mathrm{Z}_{\min }$ & 1290.78 & 0.8041 & -2.5421 & -2.5421 \\
\hline
\end{tabular}

Table 4. Collaborative workspace limit position and slave 2 joint variable parameters

\begin{tabular}{|c|c|c|c|c|}
\hline & $\begin{array}{c}\text { Extreme-po } \\
\text { sition/mm }\end{array}$ & $\theta_{2}^{1} / \mathrm{rad}$ & $\theta_{2}^{2} / \mathrm{rad}$ & $\theta_{3}^{2} / \mathrm{rad}$ \\
\hline $\mathrm{X}_{\max }$ & 2102.56 & 1.646 & -1.81 & 0.7792 \\
$\mathrm{X}_{\min }$ & 900.872 & -0.821 & 0.052 & 0.0521 \\
$\mathrm{Y}_{\max }$ & 1112.33 & 1.775 & 0.400 & 0.4004 \\
$\mathrm{Y}_{\min }$ & -1112.33 & 0.513 & -2.89 & -2.8906 \\
$\mathrm{Z}_{\max }$ & 1290.78 & 0.914 & -0.53 & -0.5308 \\
$\mathrm{Z}_{\min }$ & 1290.78 & 0.804 & -2.54 & -2.5421 \\
\hline
\end{tabular}

\section{Conclusion}

In this paper, a two-robot kinematics model is established. The workspace is solved based on Monte Carlo method, and the collaborative space boundary curve is drawn by the extreme value search domain method. The common area shape of the dual robot cooperation space and the collaborative space in space are obtained. The limit position lays a theoretical foundation for the subsequent research on the coordinated path planning of the collaborative operation of the two robots, the optimization of the robot's mechanism parameters and the cooperative collision avoidance problem.

\section{References}

1. ZHAO Daxing,LI Jiu-ling.Analysis and Simulation of Working Space of Container Painting Robot[J].Machinery Design,2008,25(2):15-17.

2. Cao Yi, Wang Shuxin. Design of Microsurgical Robot for Flexible Space[J].Robot,2005,27(5):22-225.

3. Zhou Lu. Analysis of Working Space of Dual-arm Robot Based on Monte Carlo Method[J].Journal of Mechanical Transmission,2014:85-87.

4. Lu Jun. the numerical calculation method of cooperative space of two-arm robots [J]. China Mechanical Engineering, 2001 (SI): 139-141.

5. $\mathrm{Lu} \mathrm{Ke}, \mathrm{Cao} \mathrm{Yi}$, Li Xiujuan. Solution of the cooperative space of two-arm robots[J]. Journal of Engineering Graphics, 2009: 39 43.

6. TIAN Haibo, MA Hongwei, WEI Juan. Research on working space and structural parameters of robotic arm of tandem robot[J] Journal of Agricultural Machinery, 2013, 44(4): 196-201.

7. Gupa K. C, Roth B. Design Considerations f or Manipulator Worws pace [ J ]. ASM E paper 81DET- 99, 1981, ASM E J. Of Machanical Design, 1982; 104; 704-711.
8. Cai Zixing, ed. Robotics [M]. 2 edition. Beijing: Tsinghua University Press, 2009: 54-60.

9. LI Ruifeng,MA Guoqing.Analysis of the Characteristics of Dual-arm Movement Based on Matlab Anti-human Robot[J].Journal of Huazhong University of Science and Technology Natural Science,2013,41(s1);343-347.

10. Xie Shengliang, Analysis and Simulation of the Working Space of Dual-arm Robot[J]. Mechanical Transmission, 2018, 06(29): 139-143. 